\title{
Effect of Postnatal Intermittent Hypoxia on Growth and Cardiovascular Regulation of Rat Pups
}

\author{
M.E. Pozo A. Cave Ö.A. Köroğlu D.G. Litvin R.J. Martin J. Di Fiore P. Kc
}

Division of Neonatology, Department of Pediatrics, Rainbow Babies and Children's Hospital, Case Western Reserve

University, Cleveland, Ohio, USA

\section{Key Words}

Chronic intermittent hypoxia $\cdot$ Rat pups $\cdot$ Heart rate $\cdot$ Blood pressure $\cdot$ Growth

\begin{abstract}
Background: Intermittent hypoxic episodes are common among preterm infants, although longer term consequences on growth pattern and cardiovascular regulation are unclear. Furthermore, the effects of intermittent hypoxia $(\mathrm{IH})$ may depend on the pattern of hypoxia-reoxygenation. $\mathbf{O b}$ jectives: We tested the hypothesis that a clustered versus dispersed pattern of repetitive IH during early postnatal life would induce differential long-term alteration in growth and cardiovascular regulation. Methods: Sprague-Dawley rat pups were exposed to room air or to one of two patterns of IH (clustered vs. dispersed) from 1 to 7 days of life. Body weight was measured daily for the first 8 days and weekly from weeks 2 to 8 . Blood pressure (BP) and heart rate were measured weekly from weeks 4 to 8 using a noninvasive tailcuff method for awake, nonanesthetized animals. Results: Exposure to both patterns of repetitive IH induced early growth restriction followed by later catch-up of growth to controls 3 weeks after completion of $\mathrm{IH}$ exposures. $\mathrm{IH}$-exposed rats exhibited a sustained decrease in heart rate re-
\end{abstract}

gardless of the hypoxic exposure paradigm employed. In contrast, a differential response was seen for arterial pressure; the clustered paradigm was associated with a significantly lower BP versus controls, while the pups exposed to the dispersed paradigm showed no effect on BP. Conclusion: We speculate that repetitive IH during a critical developmental window and regardless of $\mathrm{IH}$ exposure paradigm contributes to prolonged changes in sympathovagal balance of cardiovascular regulation.

Copyright $\odot 2012$ S. Karger AG, Basel

\section{Introduction}

Extremely preterm infants born at less than 28 weeks' gestation are at potential risk for later adverse health outcomes during childhood, adolescence, and adult life [1]. This has resulted in considerable interest in the longer term nutritional and cardiovascular consequences of preterm birth [2]. Previous studies have proposed a relationship between magnitude of postnatal weight gain, later hypertension, and lower insulin sensitivity [3]. The relationship between these various phenomena remains to be determined. Shorter term circulatory dysfunction has also been described in preterm versus term infants as

\section{KARGER}

Fax +41613061234 E-Mail karger@karger.ch www.karger.com
(C) 2012 S. Karger AG, Base

$1661-7800 / 12 / 1022-0107 \$ 38.00 / 0$

Accessible online at:

www.karger.com/neo
Prabha Kc, $\mathrm{PhD}$

Division of Neonatology, Department of Pediatrics, Rainbow Babies and Children's Hospital, Case Western Reserve University, RB \& C, Suite 3100, 11100 Euclid Avenue Cleveland, OH 44106-6010 (USA)

Tel. +1 216844 8452, E-Mail prabha.kc@ case.edu 
Fig. 1. Dispersed and clustered hypoxia paradigms. Dispersed hypoxia exposures consisted of 45 s of hypoxia (nadir of 5\% $\mathrm{O}_{2}$ ) followed by $4 \mathrm{~min}$ and $15 \mathrm{~s}$ of $21 \% \mathrm{O}_{2}$. These 5 min-duration cycles were presented continuously over 8 h for 7 days. Clustered hypoxic exposures consisted of $45 \mathrm{~s}$ of hypoxia (nadir of $5 \% \mathrm{O}_{2}$ ) followed by $90 \mathrm{~s}$ of $21 \% \mathrm{O}_{2}$. These $135 \mathrm{~s}$ duration cycles occurred over three periods of $72 \mathrm{~min} \mathrm{du}-$ ration per day interspersed with $2.2 \mathrm{~h}$ of RA exposure for 7 days. Both dispersed and clustered groups received 96 hypoxic episodes per day.

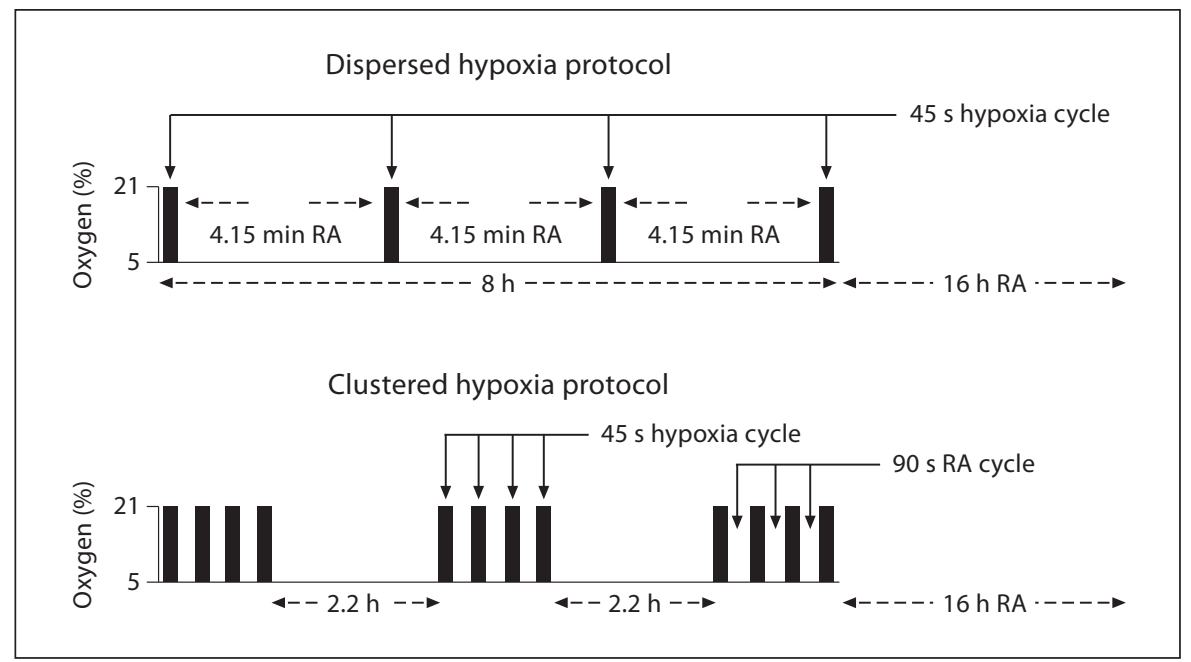

they approach a postmenstrual age of 40 weeks [4]. This was most apparent in preterm infants who developed bronchopulmonary dysplasia and manifest by a diminished pressor response and a decrease in heart rate (HR) in response to the acute stress of a $\mathrm{CO}_{2}$ exposure [4]. We, therefore, sought to explore the role of intermittent hypoxic episodes on the trajectories of both growth and cardiovascular regulation in a neonatal animal model.

Intermittent hypoxic episodes are almost universal in very low gestational age infants, and widely attributed to respiratory pauses, apnea or ineffective ventilation [5]. They may be isolated dispersed events, or occur in clusters as in periodic breathing. In both human infants and neonatal animal models there are, however, no available data comparing the effects of different patterns of intermittent hypoxia $(\mathrm{IH})$ on later morbidity apart from retinopathy of prematurity $[6,7]$. Several prior studies have employed prolonged protocols of intermittent hypoxic exposure over the first 28-30 days in neonatal rodent models [8]. Farahani et al. [8] demonstrated impaired growth with improved catch-up of weight during intermittent hypoxic exposure while Soukhova-O'Hare et al. [9] showed that prolonged exposure of neonatal pups to IH reduced vagal efferent projections in cardiac ganglia and altered baroreflex function in adult rats. As both pattern and duration of hypoxic exposure may influence the physiologic consequences of such exposure in early postnatal life, we designed this study to test the hypothesis that an initial 7-day exposure to dispersed versus clustered intermittent hypoxic episodes would differentially alter the postnatal trajectory of growth and later cardiovascular regulation in a rat pup model.

\section{Materials and Methods}

\section{Animals}

Pregnant time-dated Sprague-Dawley dams were obtained from Charles River. The rats were housed on a regular day/night cycle (lights on from 08:00 to $20: 00 \mathrm{~h}$ ) at $24-26^{\circ} \mathrm{C}$ and $40-45 \%$ relative humidity. Animals were given food and water ad libitum. All the pups were born at about the same time period. All animal experimentation was conducted in accordance with the $\mathrm{NIH}$ guidelines and approved by the Institutional Animal Care and Use Committee of Case Western Reserve University.

\section{Intermittent Hypoxic Exposure}

Litters of 10 neonatal rat pups/dam were assigned to one of three groups: (1) normoxia, (2) 96 dispersed or (3) 96 clustered hypoxic episodes per day for days of life 1-7. In a separate group, dams from room air (RA) and dispersed groups were swapped each day before the start of the exposure to determine effect of maternal stress on pups' growth. For detailed description of the exposure refer to online supplementary material (www. karger.com/doi/10.1159/000338096). Briefly, dispersed hypoxia exposures consisted of $45 \mathrm{~s}$ of hypoxia (nadir of $5 \% \mathrm{O}_{2} ; \mathrm{FiO}_{2} 60 \%$ ) followed by $4 \mathrm{~min}$ and $15 \mathrm{~s}$ of $21 \% \mathrm{O}_{2}$ (fig. 1) [10]. These $5 \mathrm{~min} \mathrm{du}-$ ration cycles were presented continuously over $8 \mathrm{~h}$ for 7 days (dispersed protocol). Clustered hypoxic exposures consisted of $45 \mathrm{~s}$ of hypoxia (nadir of $5 \% \mathrm{O}_{2}$ ) followed by $90 \mathrm{~s}$ of $21 \% \mathrm{O}_{2}$ (fig. 1). These $135 \mathrm{~s}$ duration cycles occurred over three periods of $72 \mathrm{~min}$ duration per day interspersed with $2.2 \mathrm{~h}$ of RA exposure for 7 days (clustered protocol). Overall, both dispersed and clustered groups received the same number of hypoxic episodes (i.e. 96 episodes/ day). Control animals were housed in the same chamber and were maintained at normoxia. Body weights (BW) of the pups were obtained each morning of the exposure period and weekly from weeks 2 to 8 .

Blood Pressure and HR Measurement

Arterial blood pressure (BP; systolic, diastolic, and mean BP) and HR were measured weekly from weeks 4 through 8 . Mea- 


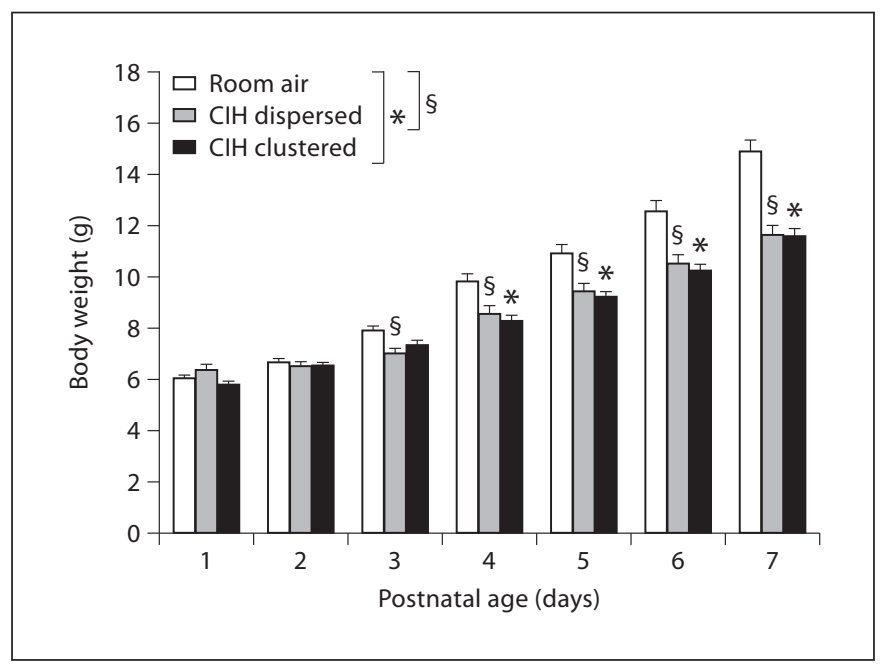

Fig. 2. Rat pups exhibited growth restriction during exposure to both dispersed and clustered IH protocols. BWs are presented from control and the two repetitive $\mathrm{IH}$-exposed groups of rats from PDs 1-7. Overall, a significantly lower BW was observed in both $\mathrm{IH}$-exposed groups as compared to age-matched control rats (clustered and dispersed vs. control: $\mathrm{p}<0.001$ ). Data are expressed as mean \pm SEM. ${ }^{*} \mathrm{p}<0.05$ for control versus clustered at designated days, and ${ }^{\S} \mathrm{p}<0.05$ for control versus dispersed at designated days. $\mathrm{CIH}=$ Chronic intermittent hypoxia.

surements were obtained using the CODA non-invasive BP system (Kent Scientific Corporation, Torrington, Conn., USA) which utilizes a volume-pressure recording tail-cuff method $[11,12]$. Awake and unsedated animals were restrained in Plexiglas holders, and were allowed to habituate for 20-30 min before taking a minimum of twenty cycles of measurements per animal. Movement at any point during the measurements was noted, and those values were excluded from analysis. HRs outside two standard deviations from the mean (suggestive of movement or stress) were excluded. Data are presented as means \pm SEM.

\section{Statistical Analyses}

A linear mixed model for repeated measures was used to assess the time course of BW, BP and HR measurements for all animals and to identify an association between these parameters and the hypoxia paradigms using SAS 5.0. A significance level of $p<0.05$ was used.

\section{Results}

\section{Body Weight}

There was a progressive increase in BW with advancing postnatal age in all groups. Repetitive IH exposure, regardless of paradigm, decreased BW throughout the

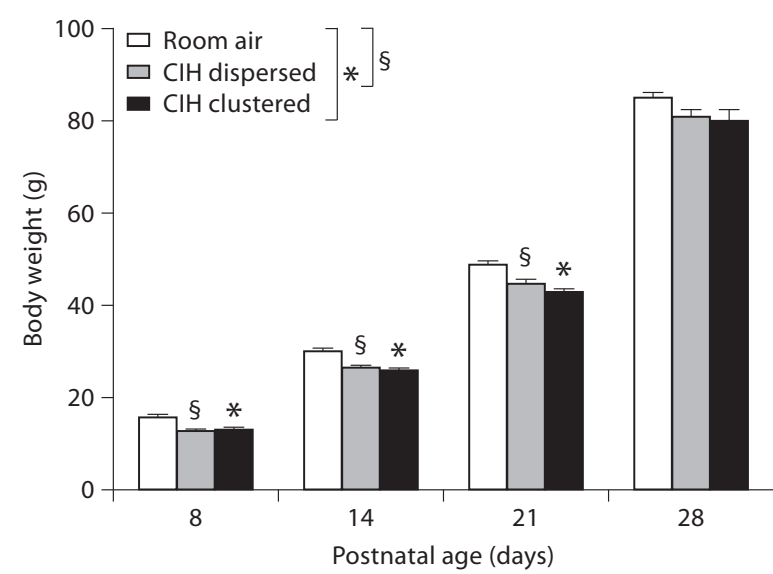

Fig. 3. Rat pups exhibited growth restriction at PD 8, 14, and 21 but not 28 after IH exposure. BWs in control and both IH groups 1, 7, 14 and 21 days following completion of IH exposures (corresponding to PDs $8,14,21$ and 28) are shown. At days 1, 7, and 14 after IH exposure, BWs of IH-exposed rats were significantly lower than control rats. A catch-up of growth was observed in both IH groups at 21 days following completion of IH exposure. Data are expressed as mean \pm SEM. ${ }^{*} \mathrm{p}<0.05$ for control versus clustered at designated days, and ${ }^{\S} \mathrm{p}<0.05$ for control versus dispersed at designated days. $\mathrm{CIH}=$ Chronic intermittent hypoxia.

exposure protocol when compared to RA controls ( $\mathrm{p}<$ 0.001; fig. 2). This decrease in BW was observed as early as day 2 and 3 of IH exposure in the dispersed and clustered paradigm, respectively, as compared to the agematched control group.

Following the 7-day IH exposure, the pups were reared in RA. At 1 (postnatal day, PD, 8), 7 (PD 14) and 14 (PD 21) days following completion of $\mathrm{IH}$ exposure, BWs in both $\mathrm{IH}$ paradigms remained significantly lower than age-matched control animals (all $\mathrm{p}<0.001$; fig. 3). However, beginning at 4 weeks of age, corresponding to 3 weeks post-IH exposure, there were no longer significant differences in weight between animal groups (postnatal week 5-8, data not shown).

In the study where dams were swapped, BW of RA pups, RA pups with swapped dams, pups exposed to dispersed protocol with swapped dams and pups with the same dam exposed to dispersed hypoxia were $18.4 \pm 0.2$, $17.8 \pm 0.3,14.6 \pm 0.3$ and $15.4 \pm 0.2 \mathrm{~g}$, respectively. The RA pups with swapped dams had slightly reduced BW than RA pups. However, there was no significant difference in the BW of pups exposed to dispersed groups with and without the swapped dams. 


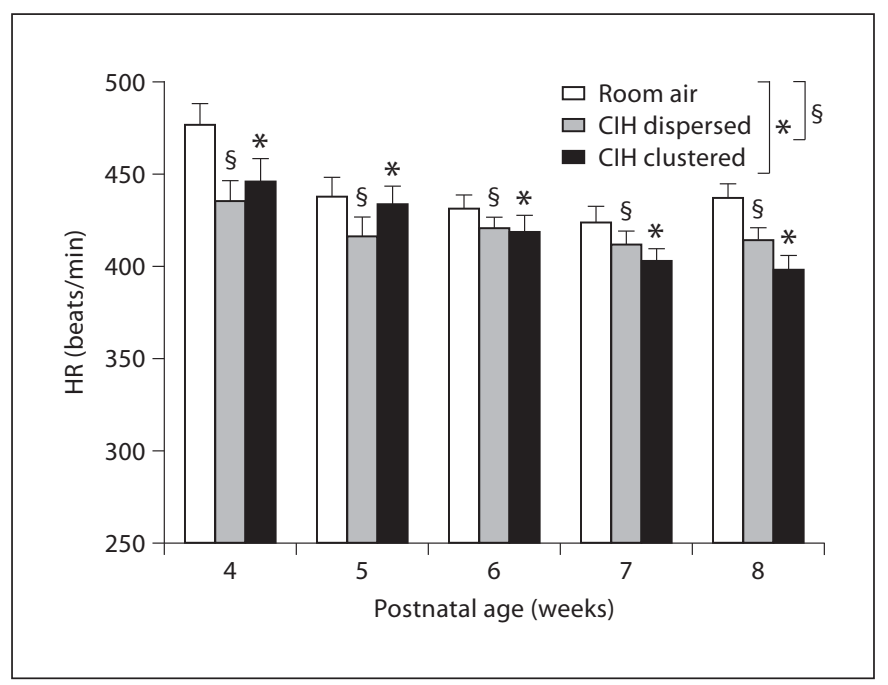

Fig. 4. Rats exhibited a decreased $H R$ after IH exposure. Resting HRs are shown in awake and unanesthetized rats during postnatal weeks 4-8. Rats exposed to the clustered as well as dispersed paradigm of $\mathrm{IH}$ during the first week of life had a significantly lower HR ( $\mathrm{p}<0.0001)$ as compared to the control rats during weeks 4-8. No significant difference was observed between the two hypoxic paradigms. Data are expressed as mean \pm SEM. ${ }^{*} \mathrm{p}<0.05$ for control versus clustered at designated days, and $\S \mathrm{p}<0.05$ for control versus dispersed at designated days. $\mathrm{CIH}=$ Chronic intermittent hypoxia.

\section{Heart Rate}

Rats exposed to the clustered and control paradigms both showed a significant decline in HR over time $(\mathrm{p}<$ 0.001 ; fig. 4) with no significant change over time in the dispersed group. Nonetheless, pups exposed to clustered as well as dispersed paradigms showed a significantly lower HR when compared to the control group ( $\mathrm{p}<$ $0.001)$.

\section{Blood Pressure}

Rats exposed to the clustered and dispersed paradigms as well as controls showed a significant increase in systolic, diastolic and mean BP from weeks 4 to 8 ( $\mathrm{p}<0.001$ ). Pups exposed to the clustered paradigm showed significantly lower systolic, diastolic and mean arterial pressure compared to controls $(\mathrm{p}<0.01)$. Systolic and mean BP data are presented in figures 5 and 6 , respectively; data for diastolic BP are not shown. In contrast, there was no significant change in systolic, diastolic and mean arterial pressure with the dispersed IH paradigm when compared to the age-matched control group. Similarly, there was no significant difference in systolic, diastolic and mean BP between the two hypoxic groups.

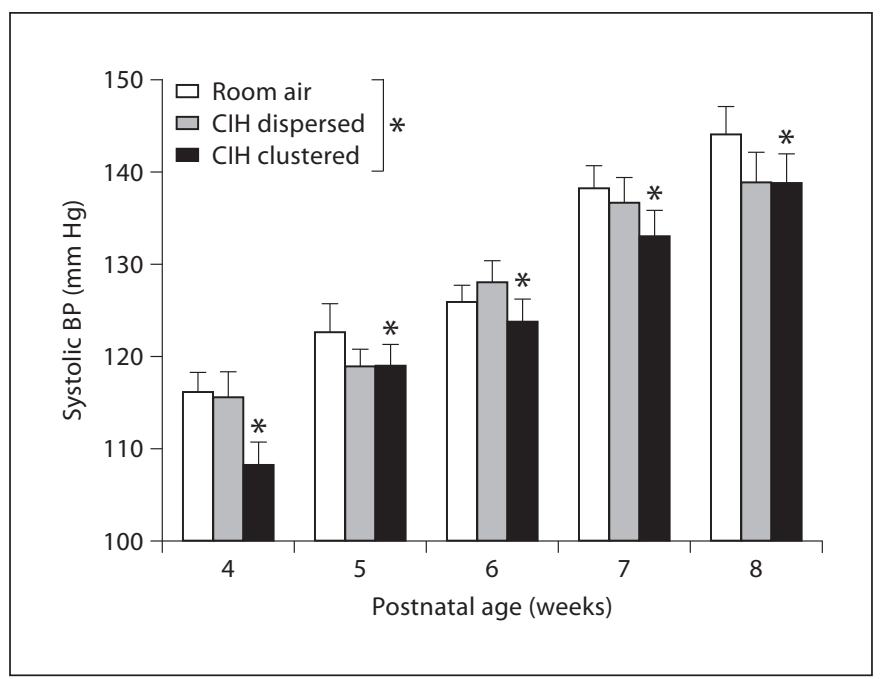

Fig. 5. Rats exhibited a differential increase in systolic BP after IH exposure. Resting systolic BP is shown in awake and unanesthetized rats during postnatal weeks 4-8. Rats exposed to the clustered paradigm during the first week of life had a significantly lower systolic $\mathrm{BP}(\mathrm{p}<0.01)$ as compared to the control rats during weeks $4-8$, whereas the dispersed paradigm group had no significant effect on systolic BP ( $p=0.3)$. No significant difference was observed between the two hypoxic paradigms. Data are expressed as mean \pm SEM. ${ }^{*} \mathrm{p}<0.05$ for control versus clustered at designated days. $\mathrm{CIH}=$ Chronic intermittent hypoxia.

\section{Discussion}

\section{Body Weight}

Exposure to repetitive IH had a significant effect on BW and resulted in growth restriction both during and after exposure to IH regardless of IH paradigm employed. While preterm infants exhibit both isolated (dispersed) respiratory pauses or apnea, and periodic breathing (clustered pauses) which manifest as IH, to our knowledge, only one prior study, focused on retinopathy of prematurity has compared the pathophysiologic consequences of these different patterns of IH exposure [7]. We have now documented a comparable pattern of growth restriction during IH regardless of exposure paradigm, and this may well be a significant contributor to postnatal growth failure in preterm infants.

Several mechanisms may contribute to this growth failure. The number of littermates per dam could affect their growth, and we therefore ensured equal number of pups in all groups. Metabolic or biochemical measurements would have revealed the severity of tissue hypoxia between the 2 different hypoxic paradigms, albeit it was beyond the scope of this paper. However, in our study the 


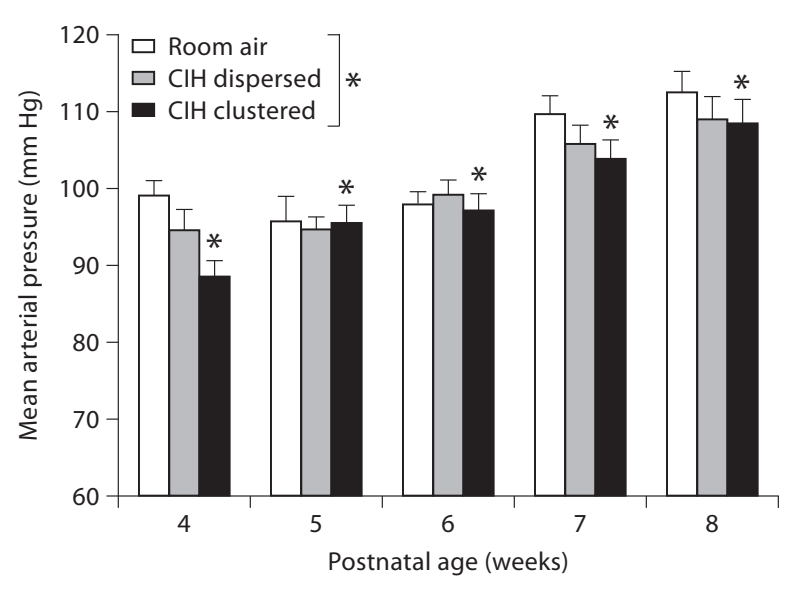

Fig. 6. Rats exhibited a differential increase in mean arterial BP after IH exposure. Resting mean arterial BP is shown in awake and unanesthetized rats during weeks $4-8$. Rats exposed to the clustered paradigm during the first week of life had a significantly lower systolic $\mathrm{BP}(\mathrm{p}<0.01)$ as compared to the control rats during weeks $4-8$, whereas the dispersed paradigm had no significant effect on systolic BP $(p=0.3)$. No significant difference was observed between the two hypoxic paradigms. Data are expressed as mean \pm SEM. ${ }^{*} \mathrm{p}<0.05$ for control versus clustered at designated days. $\mathrm{CIH}=$ Chronic intermittent hypoxia.

RA pups with swapped dams had slightly reduced BW than RA pups, which may suggest the influence of hypoxia-induced maternal stress causing reduced maternal food intake and lactation. Nonetheless, our BW data from the two dispersed groups with and without swapping of dams showed no significant difference suggesting pup growth restriction is not attributed to maternal hypoxic exposure. This finding is in agreement with previous studies $[8,13]$.

We were also interested in determining whether $\mathrm{IH}$ exposed rats gain weight at a faster rate than control animals in order to attain the same final adult weight, a phenomenon known as catch-up growth. This rapid catch-up of growth in premature infants has been hypothesized to predispose to subsequent cardiovascular risk and obesity $[14,15]$. In our study, an accelerated growth trajectory and catch-up to normoxic controls occurred by 21 days after IH exposure regardless of IH paradigm. Repetitive IH-exposed pups did not exhibit an overshoot in BW compared to their age-matched control rats. This lack of excessive weight gain following catchup growth in our adolescent rats could be related to offering regular chow to the nursing dams and their off- spring, and contrasts with the aggressive parenteral and enteral nutritional support typically provided to growing preterm infants.

\section{Heart Rate}

We have demonstrated that repetitive $\mathrm{IH}$ in the neonatal period resulted in a lower HR with advancing maturation when compared to normoxic controls. We cannot ascertain whether the decreased HR resulted from a suppression of the sympathetic nervous system or an upregulation of parasympathetic tone. Baroreflex sensitivity is considered to represent predominantly the efficacy of cardiac parasympathetic regulation. Earlier studies in mature dogs have shown that carotid body stimulation induced by hypoxia elicits bradycardia in the absence of a change in ventilation, and independent of changes in systemic BP [16]. More recently, exposure to IH has been shown to induce reactive oxygen species (ROS) which alter cell signaling mechanisms in the carotid bodies and adrenal medulla $[17,18]$. Interestingly ROS have been implicated in depressing synaptic transmission in sympathetic ganglia in mice [19]. Recent data demonstrate that prenatal nicotine evokes a defect in cardiac sympathetic innervation that is reversed by coadministration of the antioxidant vitamin C [20]. Given that $\mathrm{IH}$ is an oxidant stress, these data are consistent with our observed effect of IH on HR. Therefore, it is quite possible that $\mathrm{IH}$-induced ROS may have attenuated the sympathetic limb of the autonomic nervous system, thus contributing to a lower baseline HR after prior IH exposure.

\section{Blood Pressure}

In our study, Sprague-Dawley pups exposed to repetitive IH as well as age-matched control rats had a progressively increasing systolic, diastolic and mean BP trajectory from weeks $4-8$ after exposure. However, when compared to the age-matched control group, pups exposed to the clustered but not to the repetitive paradigm showed significantly lower systolic, diastolic and mean BP. This outcome is contrary to previous studies where SpragueDawley rats exposed to a prolonged 30-day period of postnatal IH showed no significant changes in BP [21]. There are very limited comparable data available for comparison; however, the difference in these results could be related to the IH exposure pattern and duration of exposure. In our study, we chose IH exposure duration of 7 days to more closely simulate a model of apnea of prematurity, which does not often last beyond a corrected gestational age of 44 weeks. Rats exposed during their fourth 
week of life are effectively adolescents, far beyond the age at which apnea of prematurity ceases. Critical factors that could differentially alter the effect of IH exposure might be the postnatal age at which measurements were made or the specific animal strain employed. Juvenile Wistar rats exposed to $\mathrm{IH}$ for 10 days had increased $\mathrm{BP}$ compared to control rats, however, after 15 days in normoxia, BP returned to normal levels [22]. This is in contrast to our data where clustered IH exposure occurring in the immediate postnatal period induced a sustained decrease in BP which accompanied the lower HR. Similarly, our current data are inconsistent with the increased rate of later hypertension reported in former extremely low birth weight infants $[1,2]$. This has been attributed to a combination of intrauterine growth restriction, the complications of preterm birth as well as excessive postnatal catchup growth. Based on our rat pup model, we therefore speculate that postnatal IH and accompanying growth restriction do not predispose to later hypertension. The latter in former preterm infants has recently been related to intima-media thickness rather than alteration in sympathetic vascular control [23]. Therefore, multiple potential opposing developmental changes may contribute to later cardiovascular regulation in former preterm infants.
In conclusion, our study demonstrates that repetitive IH exposure, the effect of which could have been attributed to both hypoxia and hypoxemia, during the first week of life restricted growth as early as PD 3, with subsequent recovery of BW to that of normoxia-exposed controls by 3 weeks after IH exposure. Repetitive IH exposure during early postnatal life induced a significantly lower baseline HR with advancing maturation as compared to age-matched control pups and regardless of the exposure paradigm. However, a differential effect of repetitive $\mathrm{IH}$ exposure was seen in $\mathrm{BP}$ with clustered but not dispersed IH resulting in lower pressures versus controls. We speculate that IH occurring during a critical developmental window contributes to transient growth failure and a persistent change in sympathovagal balance in our rodent model. These data provide impetus to characterize the role of postnatal desaturation/resaturation events on longer term cardiovascular and metabolic sequelae in preterm infants and explore underlying mechanisms.

\section{Acknowledgments}

This work is supported by National Heart, Lung and Blood Institute Grants 4R00HL087620 (to P. Kc) and R21HL098628 (to R. J. Martin).

\section{References}

1 Doyle LW, Anderson PJ: Adult outcome of extremely preterm infants. Pediatrics 2010; 126:342-351.

-2 Hack M, Schluchter M, Cartar L, Rahman M, Cuttler L, Borawski E: Growth of very low birth weight infants to age 20 years. Pediatrics 2003;112:e30-e38.

3 Rotteveel J, van Weissenbruch MM, Twisk JW, Delemarre-Van de Waal HA: Infant and childhood growth patterns, insulin sensitivity, and blood pressure in prematurely born young adults. Pediatrics 2008;122:313-321.

-4 Cohen G, Lagercrantz H, Katz-Salamon M: Abnormal circulatory stress responses of preterm graduates. Pediatr Res 2007;61:329334.

5 Martin RJ, Wang K, Köroğlu O, Di Fiore J, Kc $\mathrm{P}$ : Intermittent hypoxic episodes in preterm infants: do they matter? Neonatology 2011; 100:303-310.

-6 Di Fiore JM, Bloom JN, Orge F, Schutt A, Schluchter M, Cheruvu VK, et al: A higher incidence of intermittent hypoxemic episodes is associated with severe retinopathy of prematurity. J Pediatr 2010;157:69-73.
7 Coleman RJ, Beharry KD, Brock RS, AbadSantos P, Abad-Santos M, Modanlou HD: Effects of brief, clustered versus dispersed hypoxic episodes on systemic and ocular growth factors in a rat model of oxygen-induced retinopathy. Pediatr Res 2008;64:5055.

8 Farahani R, Kanaan A, Gavrialov O, Brunnert S, Douglas RM, Morcillo P, et al: Differential effects of chronic intermittent and chronic constant hypoxia on postnatal growth and development. Pediatr Pulmonol 2008;43:20-28.

\$ Soukhova-O'Hare GK, Cheng ZJ, Roberts AM, Gozal D: Postnatal intermittent hypoxia alters baroreflex function in adult rats. Am J Physiol Heart Circ Physiol 2006;290:H1157H1164.

$10 \mathrm{Kc}$ P, Balan KV, Tjoe SS, Martin RJ, Lamanna JC, Haxhiu MA, et al: Increased vasopressin transmission from the paraventricular nucleus to the rostral medulla augments cardiorespiratory outflow in chronic intermittent hypoxia-conditioned rats. J Physiol 2010;588:725-740.
11 Feng M, Whitesall S, Zhang Y, Beibel M, D’Alecy L, DiPetrillo K: Validation of volume-pressure recording tail-cuffblood pressure measurements. Am J Hypertens 2008; 21:1288-1291.

12 Malkoff J: Non-invasive blood pressure for mice and rats. Animal Lab News, Kent Scientific Corporation 2005; Nov/Dec:1-12.

13 Mortola JP, Xu LJ, Lauzon AM: Body growth, lung and heart weight, and DNA content in newborn rats exposed to different levels of chronic hypoxia. Can J Physiol Pharmacol 1990;68:1590-1594.

14 Kennedy K, Ross S, Isaacs EB, Weaver LT, Singhal A, Lucas A, et al: The 10-year followup of a randomised trial of long-chain polyunsaturated fatty acid supplementation in preterm infants: effects on growth and blood pressure. Arch Dis Child 2010;95:588-595.

15 Vohr BR, Allan W, Katz KH, Schneider KC, Ment LR: Early predictors of hypertension in prematurely born adolescents. Acta Paediatr 2010;99:1812-1818 
16 De burgh Daly M, Scott MJ: The effects of stimulation of the carotid body chemoreceptors on heart rate in the dog. J Physiol 1958; 144:148-166.

-17 Souvannakitti D, Kumar GK, Fox A, Prabhakar NR: Neonatal intermittent hypoxia leads to long-lasting facilitation of acute hypoxiaevoked catecholamine secretion from rat chromaffin cells. J Neurophysiol 2009;101: 2837-2846.

-18 Prabhakar NR, Kumar GK, Nanduri J: Intermittent hypoxia augments acute hypoxic sensing via HIF-mediated ROS. Respir Physiol Neurobiol 2010;174:230-234.
9 Campanucci V, Krishnaswamy A, Cooper E: Diabetes depresses synaptic transmission in sympathetic ganglia by inactivating $\mathrm{nAChRs}$ through a conserved intracellular cysteine residue. Neuron 2010;66:827-834.

20 Slotkin TA, Seidler FJ, Spindel ER: Prenata nicotine exposure in rhesus monkeys compromises development of brainstem and cardiac monoamine pathways involved in perinatal adaptation and sudden infant death syndrome: amelioration by vitamin C. Neurotoxicol Teratol 2011;33:431-434.
21 Sukhova GK, Nozdrachev AD, Gozal D: Neonatal intermittent hypoxia and hypertension (in Russian). Zh Evol Biokhim Fiziol 2009;45:202-207.

-22 Zoccal DB, Bonagamba LG, Paton JF, Machado BH: Sympathetic-mediated hypertension of awake juvenile rats submitted to chronic intermittent hypoxia is not linked to baroreflex dysfunction. Exp Physiol 2009; 94:972-983.

23 Hovi P, Turanlahti M, Strang-Karlsson S, Wehkalampi K, Jarvenpaa AL, Eriksson JG, et al: Intima-media thickness and flow-mediated dilatation in the Helsinki study of very low birth weight adults. Pediatrics 2011; 127:e304-e311. 